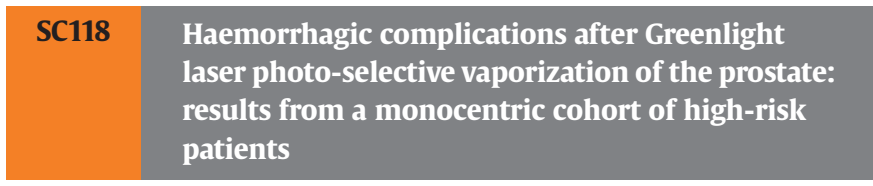

M. Sibona, P. Destefanis, A. Bisconti, A. Bosio, F. Vitiello, E. Vercelli, P. Gontero (Torino)

Introduction: Historically, BPH surgery has been associated to the risk for post - operative bleeding and transfusions. More recently, relevant technological innovations among which the Greenlight ${ }^{\circledR}$ laser, significantly improved the safety of the interventions and extended their feasibility to emergent categories of elderly, comorbid men. Anyway, addressing complex patients could still be a hard challenge. Our aim is to report a retrospective overview of a large, monocentric cohort of $\mathrm{BPH}$ patients presenting relevant comorbidities and undergoing a Greenlight ${ }^{\circledR}$ Photo - Selective Vaporization (PVP) of the prostate.

Materials and methods: We retrospectively reviewed medical records of 392 patients undergoing a Greenlight ${ }^{\circledR}$ PVP between January 2015 and December 2018 at a single centre in North - Western Italy (A.O.U. Città della Salute e della Scienza - Molinette Hospital, Torino). Baseline clinical data were retrieved. Then, an extensive analysis of all surgical complications was performed, focusing on the haemorrhagic ones. Finally, a research for clinical predictors of post - operative bleeding was performed. Statistical analysis: descriptive statistics: t-test, Pearson's chi2 test; analysis of predictors: univariable and multivariable logistic regression. Clinical variables were expressed as median (IQR) or frequencies.

Results: Median age was 72 (66-77) years. Relevant comorbidities: hypertension: 224 (57.1\%); diabetes: 63 (16.1\%); cigarette smoke: 42 (10.7\%); previous Major Acute Cardiovascular Event (MACE): 181 (46.2\%); antiplatelet therapy: 126 (32.1\%); anti - coagulant therapies: 84 (21.4\%); combined antiplatelet/anticoagulant therapies: 28 (7.1\%). A significant immediate (within 24 hours) post - operative haematuria (as severe as needing for delayed catheter removal, prolonged hospital stay or further procedures) was reported in 22 (5.6\%) patients. Severe haematuria requiring surgical revision happened in $6(1.5 \%)$ of these cases. Blood transfusions were necessary for $2(0.51 \%)$ patients. Conversely, a significant delayed (within 3 months) haematuria was reported in $52(13.3 \%)$ patients. Severe delayed haematuria happened in $10(2.5 \%)$ cases. Delayed transfusions were necessary in $3(0.77)$ patients. Having suffered of a previous MACE appeared as a significant predictor for the risk of delayed haematuria (OR 3.09, $\mathrm{p}=0.001$ ).

Conclusions: The Greenlight ${ }^{\circledR}$ laser appears extremely safe also when used in complex patients. In our cohort, although harbouring one half of very high - risk patients, we reported very low rates of immediate and delayed bleeding, with minimal risk of blood transfusions.

\begin{tabular}{|l|l|} 
SC119 & Ejaculation-sparing anatomical photo-selective \\
vaporization of the prostate (ESa-PVP): results \\
from our first monocentric series
\end{tabular}

M. Sibona, P. Destefanis, A. Bisconti, A. Bosio, F. Vitiello, E. Vercelli, P. Gontero (Torino)

Introduction: Historically, BPH surgery has been associated to ejaculatory dysfunction. More recently, several ejaculation-sparing techniques have been described. We developed a modified version of Gomez- Sancha's anatomical PVP (Ejaculation-Sparing anatomical PVP, ESa-PVP). It is based on a complete anatomical approach to the bladder neck and the veru montanum. Now, we present the results of our first series.
Materials and methods: Surgical and functional data of our first 15 patients treated with ESa - PVP were retrospectively retrieved. The interventions were performed between November 2018 and September 2019 at a single centre in North - Western Italy (A.O.U. Città della Salute e della Scienza-Molinette Hospital, Torino). Baseline clinical data were registered. Moreover, validated questionnaires scores were used to evaluate the urinary and sexual functions and health - related quality of life (IPSS, IIEF_5, MSHQ - EjD - SF, SF - 36). 6 - months follow - up data were then retrieved. Post operative standard urological outcomes and questionnaire scores were compared to baseline. Statistical analysis: Wilcoxon signed rank test, McNemar chi2 test. Descriptive statistics: median (IQR).

Results: Patients' median age was 65 (60-69) years. Median prostatic volume was 63 (46-92) cc. Ongoing alpha - blocker therapies were reported by 13 (86.7\%) patients. Baseline urological parameters: uroflowmetry peak flow: 9 (6-15.5) ml $/ \mathrm{sec}$; post - voided residual volume (PVR): 25 (8-65) cc; PSA: 2.03 (1.30-2.87) ng/ml. Baseline symptoms questionnaires: IPSS: 27 (21-31); IPSS bother score: 4; IIEF_5: 19 (14-25); SF - 36: 51 (39.8-75.8). MSHQ - EjD - SF: 13 (4-15). Globally, antegrade ejaculation was reported pre - operatively by $13 / 15$ patients (86.7\%). After 6 months, the urological parameters appeared as follows: peak flow: 18 (18-20), $\mathrm{p}=0.108$; PVR: 0 (0-2) cc, $\mathrm{p}=0.014$; PSA: $1.16(0.64-2) \mathrm{ng} / \mathrm{ml}, \mathrm{p}=0.067$. Median post - operative IPSS was 1 (0-9), with a bother score $0, \mathrm{p}<0.001$. Median IIEF_5 was $17(1-25)$, $\mathrm{p}=0.015$. Median SF - 36 was 92.9 (90.4-65.9), $\mathrm{p}=0.007$. Median MSHQ - EjD - SF score was 7 (1-13), $\mathrm{p}=0.010$, with a bother score 1. Globally, antegrade ejaculation was preserved in $9 / 15$ (60\%) of the patients, $p=0.045$. Anyway, the probability to preserve antegrade ejaculation appeared different, according to the status of pre operative ejaculatory function. In fact, antegrade ejaculation was preserved in $85.7 \%$ ( 6 out of 7 ) of those with normal pre - operative ejaculation. Conversely, it was maintained in 50\% (3/6) and 0\% (0/2) of those showing impaired or totally absent a priori antegrade ejaculation, respectively.

Conclusions: Our technique appears to be effective in preserving antegrade ejaculation, while successfully treating urinary symptoms. The status of pre - operative ejaculatory function seems to be relevant for the outcome of the procedure, being those patients with a normal pre - operative ejaculation more prone to its preservation after surgery.

\section{SC120 Effect of vacation on urinary symptoms in health care workers: an Italian multicenter study}

A. Brassetti, C. De Nunzio, A. Nacchia, R. Lombardo, A. Sica, V. Baldassarri, G. Guarnotta, Y. Al Salhi, G. Tuderti, A. Pastore, A. Carbone, G. Simone, A. Tubaro (Roma)

Introduction: Aim of our study was to assess the impact of vacation on urinary symptoms in health care workers.

Materials and methods: Between March 2018 to October 2019 a survey was carried out by enrolling health care system workers in three centers. Demographic and clinical characteristics of health care workers (i.e. age, smoking status, medical history) were collected. Urinary symptoms work related quality of life and health were assessed before and after two weeks' vacation with validated questionnaires: Overactive Bladder Questionnaire Short Form (OABqsf), nocturia episodes, Work-related Quality of Life (WRQOL) and SF-36 questionnaires. As well, NS workers (NSWs), defined as working at least one time a week from $8 \mathrm{pm}$ to $8 \mathrm{am}$, were compared to traditional workers (TWs). Wilcoxon rank test was performed to test significant differences before and after vacation. Mann Whitney test was performed to assess differences between NSWs and TWs.

Results: A total of 161 participants ( 81 males and 80 females) 41 (32/ $49 \mathrm{IQR}$ ) years old were included in the survey. A mean BMI of $28 \mathrm{Kg} / \mathrm{m} 2$ 
was computed. All subjects did not refer chronic disease or drug therapy. Overall, 71 (44\%) were NSWs with a mean of 10 hours per night and 90 (56\%) subjects were TWs with a mean of 8 hours per day. NSWs presented a higher OABq-Sf, total score (27 (IQR 23/34) vs 20 (IQR 19/24) p 0,01) when compared to TWs. Healthcare workers presented an improvement in urinary symptoms after vacation on OABq - SF (23 (IQR 20/28) vs 20 (IQR 19/24), p 0,01), WRQOL (67 (IQR $61 / 78$ ) vs 69 (IQR 61/81), p 0,01), SF36 (98 (IQR 97/100) vs 101 (IQR 99/ 102), p 0,01) questionnaries and in Nocturia defined as one event at least (39/161 (24\%) vs 29/161 (18\%), p 0.01). As well, they presented improvements in work related quality of life and overall health.

Conclusions: Vacation has a beneficial effect on urinary symptoms, work related quality of life and health in health care workers. NSWs present worst urinary symptoms when compared to TWs.

\section{SC121 Absence of Metabolic SyndRoma, preoperative urinary symptoms and flow predict positive trifecta outcome after transurethral resection of prostate: development of a clinical nomogram}

A. Nacchia, C. De Nunzio, R. Lombardo, A. Sica, S. D’Annunzio, F. Cancrini, G. Tema, V. Baldassarri, B. C. Gentile, G. Mirabile, R. Giulianelli, A. Tubaro (Roma)

Introduction: Recently, trifecta outcome has been proposed to evaluate transurethral resection of prostate outcome. Aim of our study was to evaluate the risk factors for favourable trifecta outcome in patients with lower urinary tract symptoms and benign prostatic enlargement (LUTS-BPE) undergoing transurethral resection of prostate (TURP).

Materials and methods: From 2015 onward, a consecutive series of patients with LUTS-BPE undergoing transurethral resection of prostate were prospectively enrolled. Patients were evaluated using the International Prostate Symptom Score (IPSS), uroflowmetry and transrectal ultrasound prostate volume assessment (TRUS). Metabolic syndRoma (MetS) was defined according to Adult Treatment Panel III (ATP III). Outcomes were evaluated considering the trifecta favourable outcome which was defined as a combination of the following items: (1) no perioperative complications, (2) postoperative IPSS $<8$, and (3) postoperative $\mathrm{Qmax}>15 \mathrm{ml} / \mathrm{s}$. Based on the logistic regression analysis, a nomogram was generated. Discrimination, calibration and net benefit were assessed using LROC, calibration plots and decision curve analysis.

Results: 143 patients were enrolled with a median age of 70 years (IQR 65/73), a median BMI of $25 \mathrm{~kg} / \mathrm{m}^{2}$ (IQR: 24/28) and a median PSA of $4 \mathrm{ng} / \mathrm{ml}$ (IQR 3/8). Preoperatively, median Qmax was 8 (6/10) $\mathrm{ml} / \mathrm{s}$, median IPSS was 17 (IQR 13/23) and median TRUS prostatic volume was $60 \mathrm{ml}$ (IQR: 49/82). Overall 83/143 (58\%) presented a positive trifecta outcome. Overall 55/143 (38\%) patients presented the MetS and out of them $22 / 55(40 \%)$ a positive trifecta outcome ( $\mathrm{p}=0.001)$. On multivariate analysis preoperative low IPSS (OR 0,94, CI 0.88-0.99, p 0.046), preoperative high $\operatorname{Qmax}(\mathrm{OR} 1.26$, CI 1.10-1.44, p 0.001) and absence of MetS (OR 0,21, CI 0,10-0,48, p 0.001) were independent predictors of trifecta. The predicted accuracy of the model was 0,76 . Calibration of the model was excellent $(p=0.91)$. A net benefit was recorded in the range of probabilities between 35 and $95 \%$.

Conclusions: In our study absence of Metabolic SyndRoma, symptoms and urinary flow are predictors of favourable trifecta outcome. If externally validated our nomogram may help in the preoperative counseling of patients.

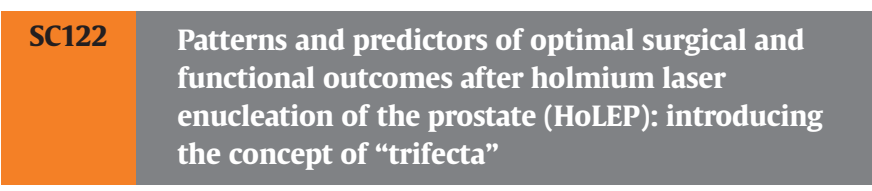

F. Sessa, R. Tellini, A. A. Grosso, M. Salvi, R. Campi, P. Verrienti, D. C. Matteo, L. Viola, F. Di Maida, S. Scelzi, A. Mari, M. Carini, A. Minervini, A. Tuccio (Firenze)

Introduction: Holmium laser enucleation of the prostate (HoLEP) is an effective option for the treatment of patients with benign prostatic hyperplasia. However, predictors of perioperative complications and treatment failure are poorly investigated. Aim of this study is to report perioperative and functional outcomes of HoLEP and to propose a trifecta as a composite outcome measure of postoperative results.

Materials and methods: Data from patients undergoing HoLEP from March 2017 to January 2019 were prospectively collected. All procedures were performed by a single surgeon. Surgical experience was divided into two consecutive surgical eras ( $<50$ vs $>50$ cases). Preoperative characteristics, including functional questionnaires [IPSS, IIEF-5, OAB-q, ICIQ-sf], and perioperative data, as well as follow-up data 3 months after surgery were recorded. Trifecta was defined as contemporary achievement of absence of $\geq$ Clavien 2 postoperative complications within 3 months, postoperative $\operatorname{Qmax}>15 \mathrm{ml} / \mathrm{s}$ and no stress urinary incontinence at 3-month evaluation. Multivariate analysis (MVA) was performed to identify predictors of failure to achieve trifecta.

Results: Overall, 137 patients were included. Trifecta was achieved in $104(75.9 \%)$ patients and was more likely to be achieved in the second ( $>50$ cases) compared to first $(<50)$ surgical era $(85.0 \%$ vs $60.0 \%$, respectively; $\mathrm{p}<0.01$ ). Preoperative features were comparable among patients with and without trifecta achievement $(p>0.05)$, except for median preoperative postvoid residual volume (PVR) that was significantly higher in the trifecta failure group (238 vs $155 \mathrm{cc}$, respectively; $\mathrm{p}=0.04$ ). Both median lasing and enucleation time were significantly longer in the trifecta failure group [38 vs $34(\mathrm{p}<0.04)$ and 55 versus 50 minutes $(p=0.009)$, respectively]. Median time to catheter removal ( 4 vs 3 days, $\mathrm{p}<0.001$ ) and hospital stay ( 5 vs 4 days, $\mathrm{p}=0.014$ ) were also significantly longer in the trifecta failure group. Early (30-days) and delayed (1-3 months) surgical complications rates were significantly higher in the trifecta failure group $51.5 \%$ and $24.2 \%$, respectively) compared to trifecta achievement group $(3.8 \%$ and $0 \% ; \mathrm{p}<0.001$ ). At 3-month follow-up, median improvement in Qmax was significantly higher in the trifecta group (14.4 vs $10.2 \mathrm{ml} / \mathrm{s}$; $\mathrm{p}=0.017$ ) while no-trifecta group reported worse median ICIQ-sf scores compared to the trifecta group ( 2 vs $0 ; p=0.002$ ). Conversely, post-operative variations in IPSS, OAB and IIEF-5 scores did not differ across two groups (all p >0.05). At MVA, surgeon experience (HR: 4.41; 95\%CI: 2.33-8.41; $\mathrm{p}<0.01$ ) and preoperative PVR (HR: 6.27; 95\%CI: $2.87-12.71 ; \mathrm{p}<0.01$ ) were significant predictors of failure to achieve trifecta.

Conclusions: In our experience, optimal outcome ("trifecta") after HoLEP was achieved in $75.9 \%$ of patients. Surgeon experience and preoperative postvoid residual volume were predictors of trifecta achievement. 